\title{
COLLATERAL RESPIRATION. TRANSFER OF AIR COLLAT- ERALLY BETWEEN PULMONARY LOBULES
}

\author{
By C. M. VAN ALlEN, G. E. LINDSKOG, and H. G. RICHTER
}

(From the Department of Surgery, Yale University School of Medicine, New Haven)

(Received for publication February 16, 1931)

During the course of some experimental work on the pathogenesis of obstructive pulmonary atelectasis we have noted a consistent occurrence of certain forms of behavior which suggests that the lobules composing single lobes of the lungs are connected collaterally with each other. Thus, after the bronchus of one lobule has been obstructed, that lobule appears to receive air from, and to give air again to, the adjacent unobstructed lobules spontaneously with the breathing. The lobule seems to be capable of a function that might be termed collateral respiration. This suggestion is, of course, contrary to the general belief that the lobular units of the bronchial tree are independent of each other at the periphery. The fact is that no certain anatomical evidence has been forthcoming, in spite of careful search by many, to show that the bronchial arborizations anastomose. To be sure, the literature contains abundant data indicating that these passages are not altogether air-tight and that when the lungs are very tensely inflated air escapes into the interstitial tissues and through the pleura and may even find entrance into the pulmonary capillaries; but this does not pertain to the situation in our experiments, where it seems that air is transferred collaterally between the lobules in the presence of normal pulmonary inflation.

Continuation of our work required solution of this question. A special group of experiments was planned to determine whether air may actually pass from lobule to lobule directly through the periphery, and, if this is so, how and when it occurs. The results of the investigation are presented here in full. ${ }^{1}$

\footnotetext{
1 Preliminary report, reference 1.
} 


\section{LITERATURE}

Evidence in favor of the existence of anastomosis between the bronchial arborizations is contained in the work of Zimmermann (2), Hansemann (3), Merkel (4), and Schulze (5). By injecting masses intrabronchially and preparing corrosion specimens, they found that the pulmonary lobules were fused together at certain points. Others who have used similar methods with attention to this matter are Flint (6), Miller (7), Laguesse and d'Hardiviller (8), and Oppel (9), but they have not obtained such connections. The studies of Professor Joseph Marshall Flint are particularly noteworthy. In summarizing his results, he states that he found "at no period in the organogenesis of the lungs openings, or fenestrae, which suggest a communication between adjacent pulmonary units. They form, as we have seen, independently at the growing ends of the tree, and as they approximate each other, it is always possible to demonstrate the interlobular or interalveolar framework without interruptions suggestive of fenestrae offering a communication between adjacent alveoli. Furthermore, in all my corrosions, many of which are complete enough to fill completely the alveoli pulmonalis and maintain the entire form of the lungs, no instance was found of an interalveolar communication." He points out, however, that the pulmonary lobules "may become compound by the loss of the interlobular septa and consequent confluence of several adjacent lobules." This work was done on pigs. Flint accounts for the appearances of interalveolar communication, which others obtained, as artifacts of extravasation of the injection masses, for high pressures are of ten necessary to drive the masses through the bronchi as far as the alveoli and delicate terminal passages are easily ruptured. The authoritative opinions of W. S. Miller (10) and Macklin (11) support this conception.

As long ago as 1733, Stephen Hales (12) showed that the lungs lose air from the surfaces when subjected to high pressures of inflation. He immersed a pair of dog's lungs in water in a bottle and then placed the whole within a negative pressure chamber, with the trachea connected by a tube to the outside. The pressure in the chamber was lowered and the lungs were gradually inflated. When the pressure reached $-69 \mathrm{~cm} . \mathrm{H}_{2} \mathrm{O}(-2 \mathrm{in}$. $\mathrm{Hg}$ ) and the lungs were tensely distended, air began to escape in streams of bubbles from numerous points in the pleural surfaces. At pressures still lower, -240 to $-275 \mathrm{~cm} . \mathrm{H}_{2} \mathrm{O}(-7$ to $-8 \mathrm{in} . \mathrm{Hg})$, the rate of escape of air was increased, but the number of points of exit in the pleura was not multiplied appreciably. This experiment has been repeated by Ewald and Kobert (13) in somewhat different form. Lungs were inflated under water simply by blowing into the trachea, and leakage was found to occur at pressures in the neighborhood of $47 \mathrm{~cm} . \mathrm{H}_{2} \mathrm{O}(35 \mathrm{~mm}$. $\mathrm{Hg}$ ). They also noticed the constancy in number of points of exit, and they added the observation that the minimum pressure of inflation required to produce leakage in a 
given specimen was the same on repeated tests. Lungs in the living animal were likewise inflated, and air was found to escape into the pleural cavity. Others $(14,15)$ have produced pneumothorax experimentally by the same procedure. Very forceful inflation was always necessary.

Bichat (16), in 1808, was the first to discover that convulsions and death were the result of inflating an animal's lungs at high pressures. Ewald and Kobert saw the same thing. Since air was found in the blood vessels at autopsy, these authors believed that the symptoms were due to air embolism and that the air entered into the pulmonary capillaries directly from the alveoli of the lungs. Joannides and Tsoulos (15) have recently studied the production of air embolism in this way. Also, they are among several investigators who have produced interstitial emphysema of the lungs and mediastinum by that maneuver.

All of these sequelae of pulmonary hyperinflation are known to occur in man. Thus, patients suffering crushing injuries of the chest without fracture of ribs often present pneumothorax or interstitial emphysema, and either or both of these effects may even be the result of voluntary straining to blow or to lift. Instances are reported in which air has passed from the lungs into the blood stream as result of raised intrapulmonary pressure. Iversen (17) found quantities of air in the circulation of persons killed by hanging, Peltauf (18) described this in persons drowned, and Lindblom (19), in new-born infants which failed to respond to strenuously applied artificial respiration. The convulsive seizures that sometimes appear with paroxysms of whooping cough have been assigned by Neubürger (20) to cerebral air embolism, and Husler and Spatz (21) furnished support for this view by demonstrating at autopsy in such cases degenerative lesions of the brain precisely similar to those produced by air embolism.

The manner of escape of air from the lungs under these circumstances is not agreed upon completely. Most authors favor a theory of alveolar rupture; indeed, widely dilated and torn air sacs have been found in the lungs in some cases (22). But the observations mentioned as to the constancy in number of the points of exit of air from the pleura and the constancy of the minimum pressure of inflation required to produce escape of air by that path have led many to believe that the escape is through openings in the alveolar walls which exist normally and are open only when the alveoli are stretched by hyperinflation. They reason that if tears in the tissues were responsible, repeated tests would show leakage at lower pressures than at first, and that the use of greater forces of inflation would multiply the number of openings.

Minute openings are well known to exist in the walls of the pulmonary alveoli in certain pathological states. In pneumonia, strands of fibrin may be seen in histological preparations to pass from alveolus to alveolus through pin-point apertures in the walls between, and these are referred to as the pores of Kohn (23). They have been described also in pulmonary edema 
(10), although made out with difficulty because of the lack of fibrin to indicate their positions. The pores are believed by many authors $(24,25,26$, $27,28,29,30)$ to be artifacts of fixation in histological preparation, or lesions. W. S. Miller (10) has described the process of their formation in pulmonary disease, claiming that in each case the opening is formed in the interalveolar septum after necrosis of two epithelial cells lying opposite each other. Others $(31,32,33,34,35,36,37,38,39,40,41,42,43)$ consider that the pores exist normally. Ogawa (44) gives good evidence of this in the results of his extensive studies of the minute anatomy of mammalian lungs. His histological methods permit the alveolar septa to be viewed in face as well as in cross-section, and the pores are seen as oval openings in the surfaces, with smooth borders formed by the epithelial cells lining the alveoli. Ogawa itates that the pores are readily distinguishable from artificial defects, for the latter present irregular and jagged borders and pass through, rather than between, the epithelial cells. The work was performed entirely upon normal lungs and includes a large variety of species.

\section{EXPERIMENTAL MATERIALS AND METHODS}

Lungs removed from the body were used for a part of the experiments, including specimens from man, dog, cat, rabbit, calf and pig. In the remainder of the experiments, lungs in the living animal were employed; and here dogs were the subject exclusively, because the endobronchial instruments at hand were adapted only to that species.

Experiments in vitro. The lungs were obtained and used immediately after death, with the exception of those from man, which were secured at autopsy from 8 to 20 hours after death. They were examined carefully for the presence of lesions and peculiarities of structure, and then a unit of one or more lobes was detached at a time and used for experimentation. Each lobe was prepared by tying a cannula into the stem bronchus or by similar treatment of one or more of the main bronchial branches. In doing the latter, it was necessary to dissect away the connective tissue at the hilus enough to expose ${ }_{0}$ and pass a ligature about each branch to be cannulated, and this was carried out with the utmost care to avoid injury to the parenchyma of the lobe. The majority of these experiments entailed injection of one cannula with air under delicately controlled grades of pressure. The apparatus for this consisted of a rubber tube connecting the bronchial cannula with a source of compressed air (tap from the main of the system of compressed air in the building) and two tubes branching from this, 
one of which led to a manometer and the other of which ended shortly by immersion in a glass of water. The second of these branching tubes served to regulate the flow of air to the specimen. Sufficient current of air was turned on at the tap to be slightly in excess of that needed for inflation of the specimen, the excess air was allowed to escape constantly from the submerged tube, and by raising or lowering that tube in the water the flow to the specimen was regulated. While the injection was going on, the cannula of another bronchus was watched for the escape of air, and this was facilitated by immersing the free end of the cannula just under the surface of water in a separate dish. Some of the experiments required that the lung be expanded during the injection. The specimen was placed for this purpose in a negative pressure chamber with the cannulas extending to the outside, and the pressure within the chamber was lowered until the desired degree of expansion was obtained. The cannulas were then connected with the injecting apparatus and the test was made as described. The complete arrangement is illustrated in Figure 1.

Experiments in vivo. The dogs were prepared by hypodermic injection of morphine and atropine and were anesthetized by inhalation of ether. The neck was incised in the ventral mid-line, and the trachea was delivered and divided completely just below the larynx. The distal end of the trachea was fitted with a short glass sleeve of the same diameter, to hold wide the entrance for instrumentation and to control persistent oozing of blood. From this time on, the anesthesia was maintained by continuous intratracheal insufflation of ether-vapor and air. A bronchoscope was introduced through the tracheotomy opening, and one bronchus was chosen for cannulation, the position and depth of its orifice being determined exactly. The bronchus chosen was either the main bronchus of the right lower lobe, at a point just distal to the origin of the first branch of that lobe, or the bronchus supplying the right middle, lower and accessory lobes, or the bronchus supplying the right lower and accessory lobes. Thus, the first choice included one part of the bronchial tree of one lobe, while the other two included the entire bronchial trees of two or three lobes. The bronchoscope was then removed and a long cannula was inserted in its place and fixed at the chosen point. A cannula was designed especially 


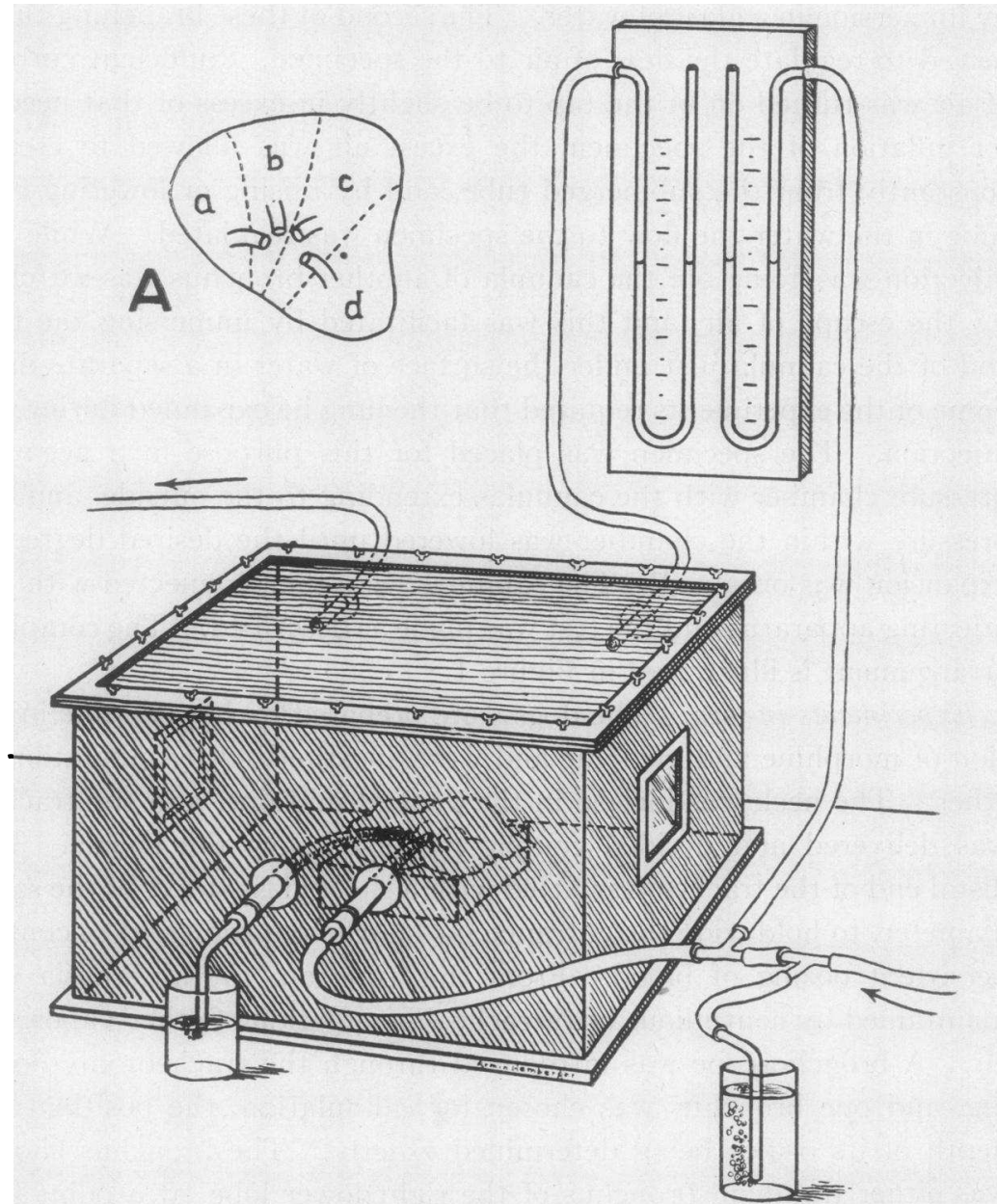

Fig. 1. Apparatus for Measuring Resistance to Collateral Transfer of Air in the Excised Lungs

Center: negative pressure chamber, containing one lung lobe. Two cannulas from the bronchi extend to the outside. Right, below: system of tubes for injection of air. Left, below: glass of water to detect escape of air. Right, above: manometers. A: diagram of lung lobe. 
for this purpose (45) ${ }^{2}$ (Fig. 2). One end could be dilated after introduction by revolving a cap at the other end. The dilated end then presented a narrow ring of metal and rubber for selective attachment to the bronchial wall. The animal was sacrificed and autopsied after the experiment in every instance, and the position and security of the cannula were determined. It was invariably found that the attachment resisted vigorous twisting and pulling and that it showed no leakage of air under test with very high pressures. In many experiments, the cannula was provided with a valve for control of air respired through it. The valve was a bottle with two necks, partly filled with water. Two glass tubes entered the bottle through tight corks, one extending just beneath the surface of the water, and the other terminating above the water. The cannula was connected by a rubber tube to the first of these when it was desired to permit only expiration through the cannula, and it was connected to the second when inspiration only was desired. See Figure 3, A and B.

Manometric measurements were made with water as medium, since the pressures encountered were generally very small.

Six objectives were dealt with in this work; therefore the experiments will be presented in that number of sections. For the sake of cogency, each section will be treated separately by introducing it with statement of object and closing it with resumé of deductions.

\section{EXPERIMENTS}

\section{Section 1}

Object: To test the air-tightness of the pulmonary lobule, seeking for transfer of air collatera!ly from its airways into those of adjacent lobules.

Experiments in vitro, in man, dog, cat, rabbit, calf, pig.

Protocol 1. Dog's lungs. The bronchus of one lobule and that of one lobe injected in turn with air; the inflation of the lobule and of the lobe and the paths taken by the air observed. A dog's lungs were procured and examined. The

${ }^{2}$ Trial was made of the type of bronchial cannula employed by others $(46,47,48)$, which has an inflatable rubber collar at one end to secure it in the bronchus. This proved unsuitable for the purpose at hand, for, when the collar was inflated, it elongated within the bronchial lumen and covered too great a length of the wall. Also, the rubber slipped easily and could not be depended upon to maintain a given position. 


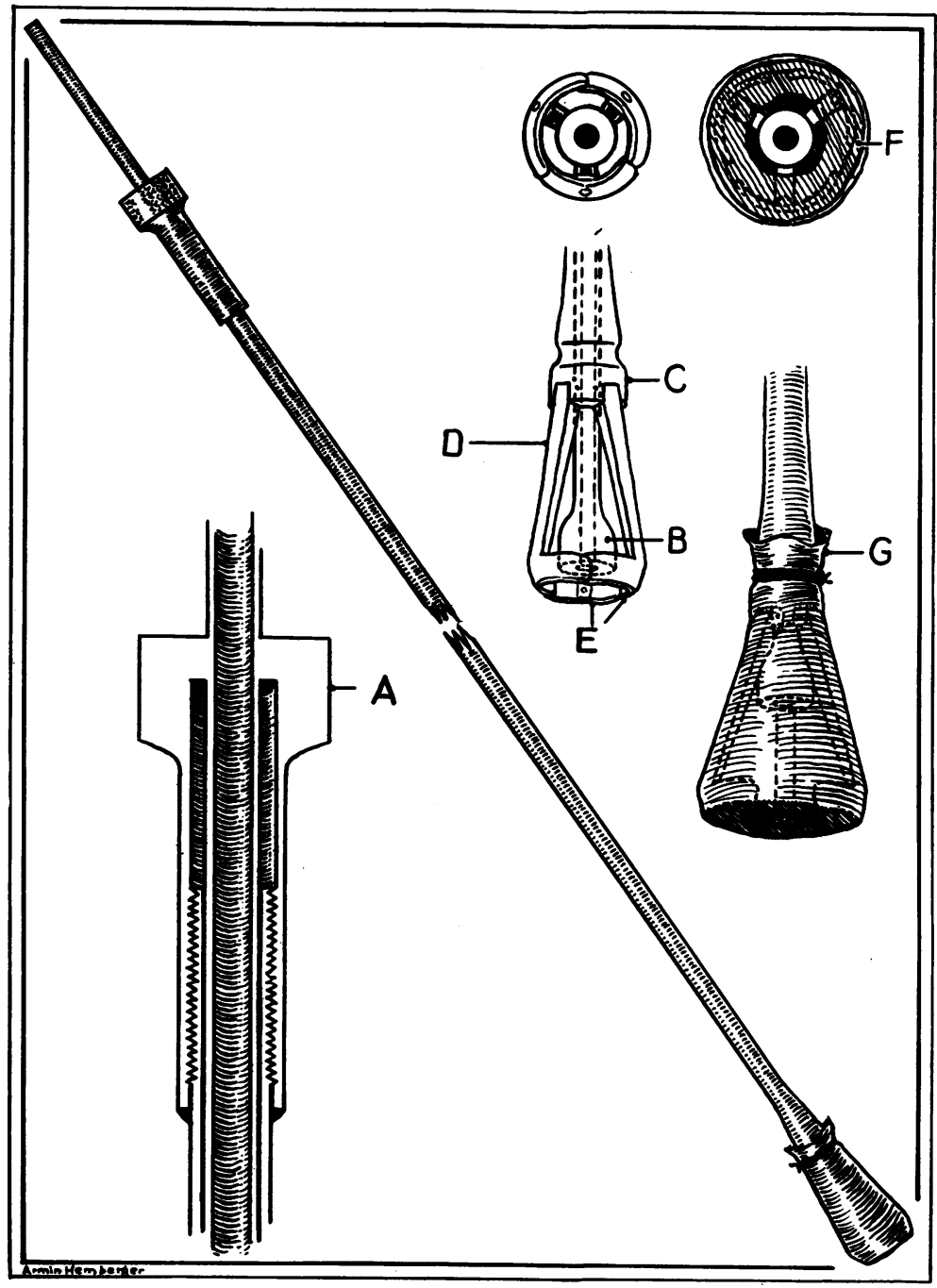

Fig. 2. Dilatable Bronchial Cannula

Center: the cannula. Above: detail of the dilatable end. Below: detail of the reverse end. The screw-cap is revolved to produce dilatation. 


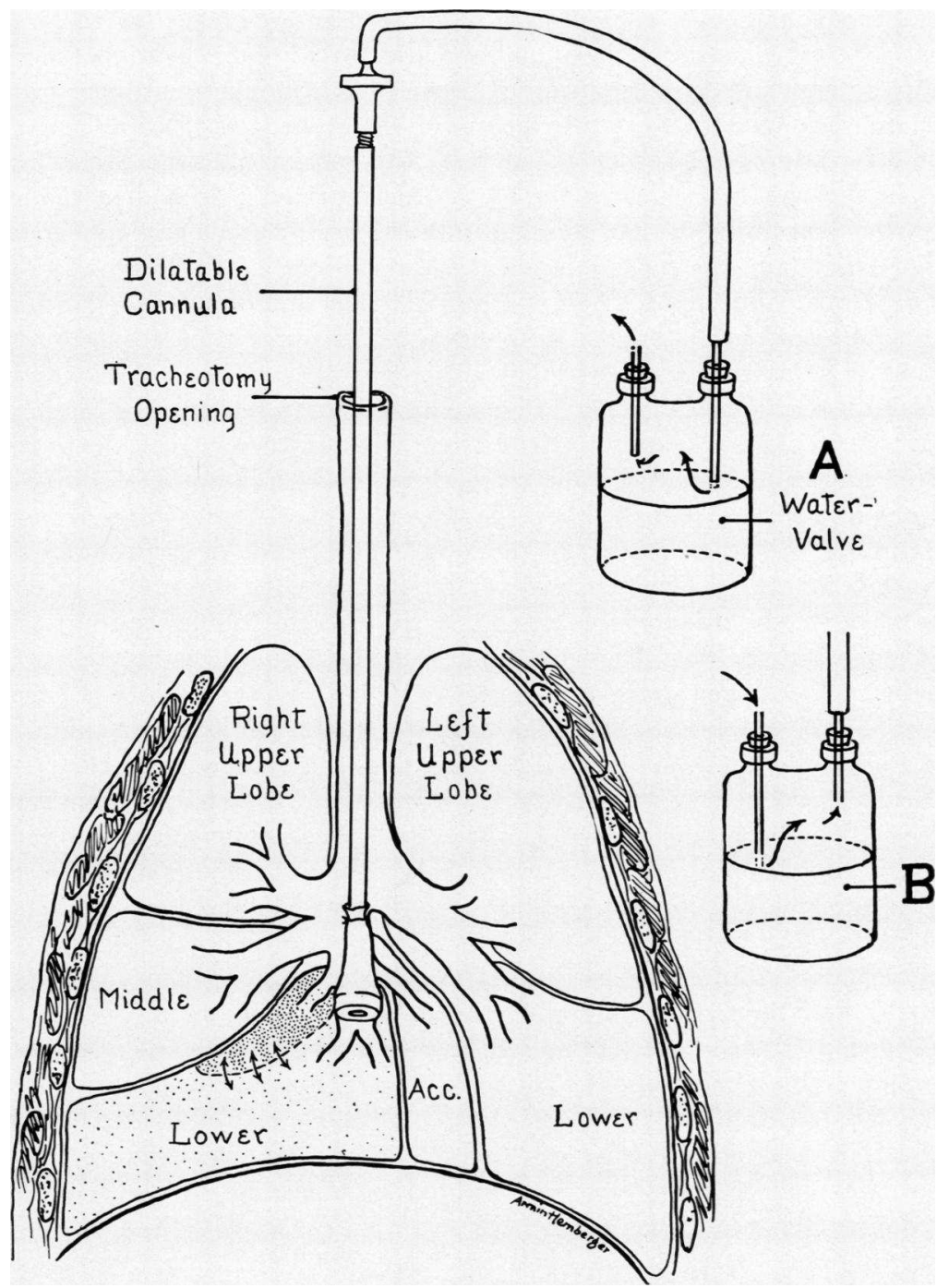

Fig. 3. Apparatus for Detecting Collateral Respiration in the Living Dog

Center: trachea and lungs, with dilatable cannula fixed in one bronchus of the right lower lobe. Above: connection to water-valve. A: watervalve, arranged to permit expiration only. B: water-valve, arranged to permit inspiration only. Arrows: path of collateral respiration between the obstructed part (lightly dotted) and the free part (densely dotted) of the cannulated lobe. 
lobes were found to be entirely separated by fissures, except for very small areas of confluence near the hilus, and there they were held together by complete septa of loose connective tissue. The lobular markings on the surfaces were indistinct or quite lacking. The right lower lobe was selected first for testing and was detached from the specimen. One cannula was tied in the first branch from the stem bronchus and a second in the stem bronchus just distal to that point, so that the first cannula supplied a single major lobule and the second supplied the remainder of the lobe. The first cannula was connected to the apparatus for injection of air, and the second was submerged in water. A slow current of air was then turned on and regulated so as to inflate the lobule very gradually. The lobule was seen to enlarge symmetrically, and it soon showed scattered patches of cortical inflation. When the expansion was about three-fourths normal, the progress halted and air began to escape from the second cannula in a continuous stream of bubbles, without there having been the slightest appearance of inflation of the other portion of the lobe. The escape of air was observed for a few minutes and no change was noted, and then the rate of injection was made steadily to increase. As a result of this the lobule expanded more and more and the flow of air from the second cannula became greater and greater. When the expansion had reached a degree that was about normal, the rate of injection was reduced and regulated so as to maintain that state, and the specimen was submerged in water. No air escaped from the pleura. Then the specimen was taken from the water and the second cannula was closed. The portion of the lobe supplied by it began immediately to inflate and continued to do so until it was fully distended. It presented the same appearances of inflation as were shown by the single lobule, i.e., symmetrical expansion. At no time was interstitial extravasation of air seen in the plane of fusion of the two divisions of the lobe or at other points. The first cannula was then closed, stopping the air current, and the second cannula was opened. The lobe collapsed rapidly as a whole, but when air had ceased to escape, the lobule supplied by the first cannula remained very slightly more inflated than the rest of the lobe. The lobe was discarded, and the right upper and middle lobes were detached from the specimen for testing. They were allowed to remain connected together, for they presented a maximum degree of confluence (over an area of about $1 \mathrm{sq} . \mathrm{cm}$.). The stem bronchus of each lobe was fitted with a cannula. That of the right upper lobe was connected with the apparatus and injected with air, while the cannula of the other lobe was submerged in water and watched for the escape of air, just as in the preceding experiment. The inflation of the first lobe was carried far beyond the normal degree of expansion and to a point when air escaped audibly from the pleural surfaces, but no air bubbled from the second cannula.

These experiments were repeated with many other specimens of dogs' lungs, and the results were always similar. The lungs of cats were used in 
the same way. Their gross anatomical characteristics and their behavior to lobular and lobar inflation were quite similar to those of the dogs' lungs and do not require separate description.

Protocol 2. Dog's lungs-one atelectatic lobe. The bronchus of one lobule injected with air; the inflation of the lobule and the path taken by the air observed roentgenographically. The accessory lobe of a dog's lungs was obtained. It had been rendered atelectatic and was shrunken and uniformly airless. ${ }^{3}$ The lobe was bilobular, suggesting somewhat the shape of a butterfly; and the stem bronchus bifurcated at the hilus into branches of equal size for the two wing-like major lobules. A cannula was tied into each of the two branches, and they were connected with apparatus for injection of air. A roentgenogram was then taken of the specimen and adjacent connections. This is reproduced in Figure 4, at A, and shows the lobe as a shadow of homogeneous consistency. The cannula for injection is represented at $a$, the exhaust tube for controlling the injection is shown at $b$, and the second cannula is at $c$, with its free end submerged in water in a small dish at $d$. Injection was begun and one lobule was inflated gradually until air began to escape from the cannula of the other; and with the air continuously bubbling through the water a second roentgenogram was taken. See Figure 4, B. Here, at $e$, the aerated portion of the lobe is represented as an area of rarefaction, including the greater part of the first lobule and a narrow zone at the border of the second. At $f$ appears a circular area of rarefaction betraying the escape of air through the water. The second cannula was then closed by turning a stop-cock, to retain the air entirely in the specimen, and the progress of inflation that ensued was followed in a series of roentgenograms. See Figure 4, C, D, E and F. This shows that the air spread to the bronchi of the second lobule, at $g$, and to the parenchyma of both lobuies, until all parts of the lobe were flled with air.

This experiment was performed with one other specimen, and similar results were obtained.

Protocol 3. Lungs of man. The bronchi of several lobules injected in turn with air; the inflation of the lobules and the paths taken by the air observed. The left lung was obtained from a young man who had died a few minutes after receiving abdominal and cerebral injuries. The two lobes were confluent over about one-half of their opposed surfaces, and in the plane of confluence was a complete septum of loosely packed connective tissue. Lobular markings were indistinct for the most part. The lobes were allowed to remain attached together, and they were prepared for testing by cannulating the three main branches of the stem bronchus of each lobe. Two branches were chosen for use at first, the lobules of which lay adjacent, and

${ }^{3}$ The atelectasis was produced by obstructing the stem bronchus of the lobe with wax 3 days before sacrifice $(49,50)$. The wax was removed at autopsy before the lobe was used. 


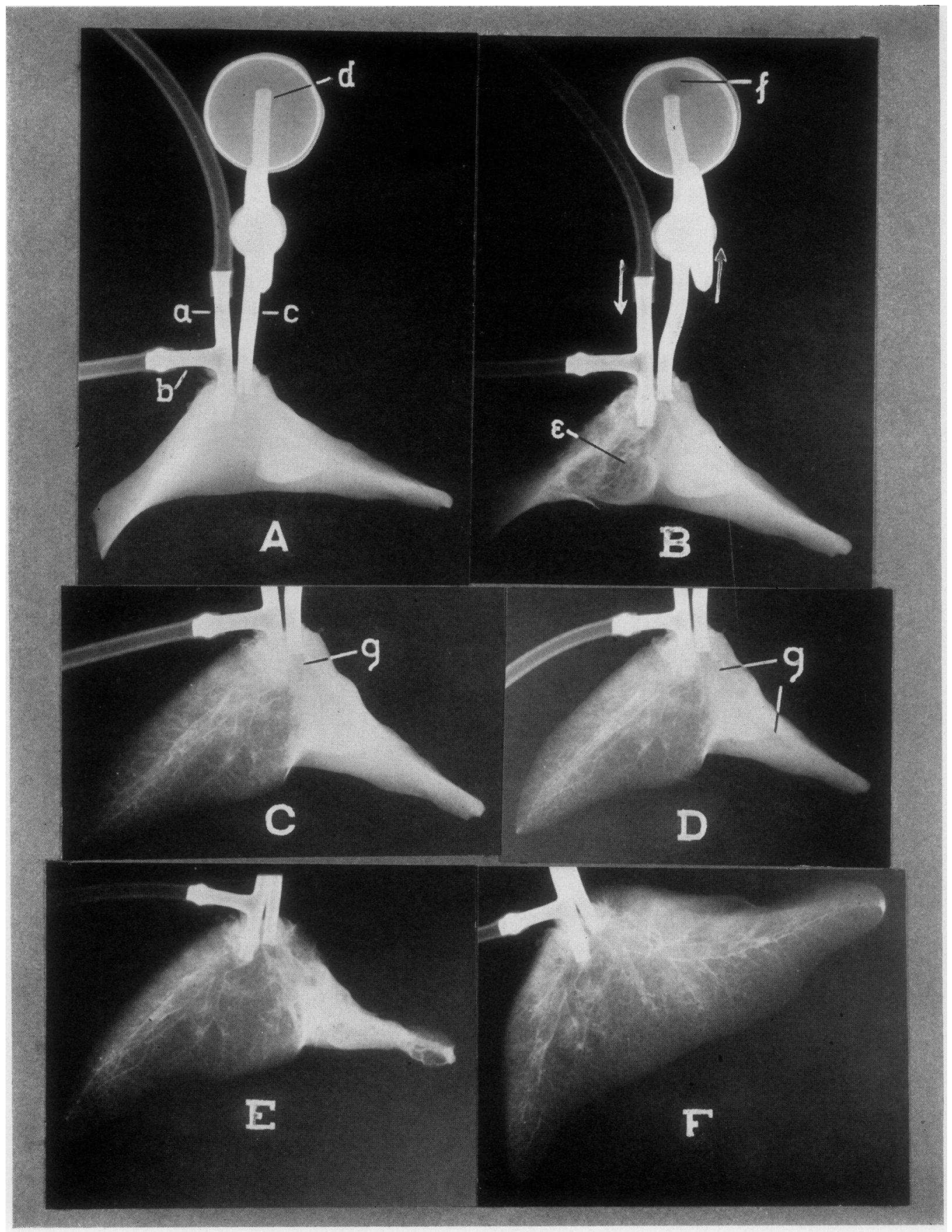

Fig. 4. Series of Roentgenograms of a Lobe of Dog's Lungs, Made While Air Was Being Injected into One Bronchial Branch

A: lobe fully collapsed. B-E: stages of inflation, showing collateral transfer of air from one major lobule $(e)$ to the second $(g)$, with escape $(f)$ from the bronchus of the latter. F: lobe fully inflated. 
the cannulas of the others were closed. Connections were made and air was injected into one, while the other was watched for escape of air. The lobule of the first became inflated to about one-fourth normal expansion, when air began to flow from the cannula of the second. The flow was continuous and kept pace with the injection, the expansion of the lobule remaining constant. The rate of injection was then increased. The lobule expanded farther and farther, and when the expansion had reached about a normal degree, the escape of air from the second cannula had increased to an exceedingly rapid flow. There was no loss of air from the pleural surfaces nor appearance of interstitial emphysema. The cannulas were then disconnected from the apparatus and, after the air had escaped from the lobule, they were closed. The test was applied to two other lobules which lay adjacent to each other and in the other lobe. The result was the same. Again, a pair of lobules was chosen which lay adjacent to each other but in different lobes (with the interlobar septum between), and the test was applied. This time the inflation of one lobule had to be carried distinctly beyond normal before air escaped from the cannula of the other.

Another normal human lung was tested in this way. The interlobar fissure was deeper in this case and the area of confluence was proportionately less extensive. The plane of contact was occupied by a complete septum of connective tissue, as in the other specimen. The results were similar in every respect, except that when two lobules were tested which lay adjacent to each other and in different lobes, there was no transfer of air, even when inflation of one was carried far beyond normal, with loss of air from the pleural surfaces and interstitial extravasation.

Protocol 4. Rabbit's lungs. The stem bronchus of tiio lobes injected in turn with air; the inflation of the lobes and the paths taken by the air observed. The lungs were secured from a large rabbit. The lobes were found to be widely confluent and the interlobar septa were thin and incomplete. Lobular markings were lacking. The stem bronchi were so imbedded in the lung that it proved impossible to expose the first branches for cannulation without injuring the parenchyma, so the experiment had to be limited to dealing with whole lobes. The middle and lower lobes on the right were selected for the first test, and, leaving them attached to the lungs, the stem bronchus of each was fitted with a cannula. The cannula of the middle lobe was connected to the apparatus for injection, and that of the lower lobe was closed. Injection was begun. The middle lobe inflated to a point which represented about one-half normal expansion when the other lobe began to expand, and then the process continued in both to about three-fourths normal expansion. At this point it ceased and air could be heard to escape from the pleural surfaces of both lobes. (Pressure of injection, $20 \mathrm{~cm} . \mathrm{H}_{2} \mathrm{O}$.) The left upper and lower lobes were tested in the same manner and similar results were obtained. In neither case was interstitial emphysema seen.

Protocol 5. Calf's lungs. The bronchi of several lobules injected in turn 
with air; the inflation of the lobules and the paths taken by the air observed. The lungs of a calf were secured. The lobes were found to be separated almost entirely by fissures, and thick septa occupied the small planes of interlobar confluence that existed. The lobular markings were very pronounced. By dissecting with blunt instruments between the lobules, it was discovered that the septa lying in that position were much heavier than those seen in the lungs of the other animals examined, and that between the major lobules they extended throughout, from hilus to periphery. The septa between the minor lobules separated only the peripheral parts, and in the deeper parts the lobules were intimately fused together. The right lower lobe was first chosen for testing and was detached from the lungs. The three main branches of the stem bronchus were fitted with cannulas. Two adjacent branches were used first, and the other was closed. One of the pair was connected with the apparatus for injection, and its lobule was inflated. The expansion reached an approximately normal degree without any air escaping from the cannula of the second lobule. Inflation was carried further, and soon air was heard plainly to leak from the pleural surfaces. With more inflation, air began to extravasate into the septum between the lobules, to split the two lobules apart, and to form blebs under the pleura. Still no air passed from the cannula of the second lobule. The apparatus was now disconnected and the lobule was allowed to collapse. The cannula of the third lobule was connected for injection, that of the second was submerged in water to be watched for escape of air, and that of the first was closed. The second and third lobules were adjacent. The results of the injection were the same as before. Attempts were then made to apply the test to minor lobules, but the bronchi supplying them were too small to be cannulated. The right upper lobe was next taken from the specimen of lungs and tested, and the results were no different than before.

The lungs of a pig were obtained and examined. They presented interlobar and interlobular septa as heavy and extensive as those found in the lungs of the calf. The results of experiments with inflation of lobules were very similar to those described in the protocol above.

\section{Deductions}

1. The air passages of lobular divisions of excised lungs are not airtight at the periphery. When the inflation of one lobule alone is increased, the air may escape by one or more of three courses besides that through the bronchus of the lobule, viz., into the air passages of neighboring lobules, through the pleura, and into the interstitial tissues. The first of these courses is undertaken when the inflation of the lobule is increased moderately and within normal limits, while the other two courses are assumed only with over-inflation of moderate 
or extreme degrees. An exception to this occurs with rabbit's lungs, for the second course is undertaken at degrees of inflation only slightly greater than those at which the first course is assumed. The first course, namely, collateral transfer between lobules, depends also upon intimate interlobular fusion, and this circumstance varies with the species of animal and with the regions of the lungs. as follows: In man, dog and cat, lobules in the same lobe are intimately fused, and lobes are separated completely by fissures and thick septa; and in these species air passes freely from. lobule to lobule when both of them are contained in the same lobe, but not when they are contained in different lobes and lie adjacent. The rabbit presents intimate fusion between lobules and between lobes, and air passes freely from lobule to adjacent lobule whether the lobules lie in the same, or in adjacent lobes. The calf and pig have thick septa and fissures which completely separate the lobes, thick septa which completely separate the major lobules, and thinner septa which only partly separate the minor lobules; and in these species air fails to pass between major lobules in any position. Passage between minor lobules could not be tested for, but that there was provision for such passage was evident from the intimate interlobular fusion that existed.

2. Collateral transfer of air between the bronchial passages of adjacent pulmonary lobules probably occurs by way of the fine arborizations at the plane of interlobular fusion, since it occurs only when the air extends to the periphery. Moreover, the transfer seems to be direct and without traversing connective tissues; because, first, interstitial emphysema does not appear in the region where the transfer occurs; second, wherever the transfer fails and interstitial emphysema develops from over-inflation the transfer is not initiated thereby; and, third, the pattern of expansion produced in one lobule by transfer of air from another is symmetrical and exactly the same as that of a lobule which is inflated by injection into the bronchial tree.

Section 2

Object: To test the air-tightness of the pulmonary lobule, seeking for transfer of air collaterally into its bronchial arborizations from those of adjacent lobules.

Experiments in vivo, in the dog.

Protocol 6. Living dog's lungs. The bronchus of a division of lobules in one 
lobe and that of three entire lobes aspirated in turn, to exhaust the air from those parts of the lungs; the yield of air measured and compared with the estimated capacities of those parts for air; the effects of the aspiration upon intrabronchial pressures observed. A dog was anesthetized and tracheotomized. The dilatable cannula was introduced through the tracheotomy opening and fixed in the bronchial tree of the right lower lobe, at a point just distal to the origin of the first branch of the lobe. The cannula was connected to a manometer and the intrabronchial pressures were read (respirations light). They were: inspiration, $-2.2 \mathrm{~cm} . \mathrm{H}_{2} \mathrm{O}$, and expiration, $2.5 \mathrm{~cm} . \mathrm{H}_{2} \mathrm{O}$. A syringe was then connected in place of the manometer, and air was aspirated from the cannula at the rate of $100 \mathrm{cc}$. per minute. The yield of air was free at all times, and when $500 \mathrm{cc}$., which was at least 5 times the amount of air normally contained in the part of the lobe cannulated, had been removed without indication of exhausting the supply, aspiration was stopped and the syringe was replaced by the manometer. The pressures were: inspiration, $-2.0 \mathrm{~cm}$. $\mathrm{H}_{2} \mathrm{O}$, and expiration, $2.5 \mathrm{~cm} . \mathrm{H}_{2} \mathrm{O}$. Next, the cannula was loosened from the bronchus, withdrawn slightly and fixed again at a point in the right primary bronchus, so as to include in the cannulation the whole of the middle, lower and accessory lobes. The syringe was connected with the cannula once more and air was aspirated at the rate of $100 \mathrm{cc}$. per minute. With removal of $125 \mathrm{cc}$., resistance to aspiration increased perceptibly and continued to do so as more air was removed, until at $200 \mathrm{cc}$., which was less than the amount normally contained in the three lobes and about equal to the amount which they would be expected to yield on collapsing, no more could be withdrawn. The intrabronchial pressure at that time proved far too low to be measured by the manometer.

This experiment was repeated in several other dogs and similar results were obtained. The observation was added that aspiration of small amounts of air, as small as $3 \mathrm{cc}$., from one or more whole lobes resulted in distinct and lasting depression of the intrabronchial pressures. This effect was always in contrast to that of aspiration from lobular divisions of single lobes, where removal of air in any quantity failed to change the pressures.

\section{Deductions}

3. The air passages of lobular divisions of the lungs in living dogs are not air-tight at the periphery. When the inflation of one lobular division alone is decreased, air may enter freely by another way than that of the bronchus of the lobule. The portal of entrance is probably the collateral passage, already referred to, which connects the airways of adjacent lobules. The collateral transfer occurs so freely as completely to restore the inflation of the lobular division in question.

4. Collateral transfer of air does not take place from lobule to lobule 
when the lobules lie adjacent and in different lobes of the lungs of living dogs.

\section{Section 3}

Object: To test for collateral respiration on the part of the pulmonary lobule with obstructed bronchus.

Experiments in vivo, in the dog.

Protocol 7. Living dog's lungs. The bronchus of a division of lobules in one lobe and that of two entire lobes obstructed in turn with a valve, to allow only expiration from those parts of the lungs; the total amounts of air expired past the valve compared to the estimated capacities of those parts for air; the expired air analyzed; the valve reversed, and the total amounts of air inspired past the valve compared to the estimated tidal air of those parts. A dog was anesthetized and tracheotomized. The dilatable cannula was introduced and fixed in the bronchial tree of the right lower lobe, at a point just distal to the origin of the first branch of the lobe. The cannula was connected to the submerged tube of the water-valve, Figure 3, A. Now, with the first expiration air bubbled freely from the submerged tube, and with the following inspiration bubbling ceased and a column of water was sucked up in the tube about 5 $\mathrm{cm}$. The second expiration was accompanied by a like discharge of air, and the inspiration produced the same elevation of water; and these effects accompanied each respiratory cycle throughout an half-hour period of observation. The total volume of air which escaped during that period was obviously many times that of the capacity of the division of the lobe from which it came. The bronchial cannula was then disconnected from the valve, and free respiration was permitted to take place through the cannula for a few minutes. The cannula was reconnected to the valve, but this time to the elevated tube, Figure 3, B. Now, with the first expiration a column of water rose about $5 \mathrm{~cm}$. in the submerged tube of the valve, and with the following inspiration air bubbled freely from that tube. The second, and every other, respiration in an half-hour period of observation produced the same effects. The dog gave no sign of respiratory embarrassment. The total volume of air which entered the cannula was obviously enormously greater than the capacity of the cannulated division of the lobe to receive air. Next, the cannula was disconnected from the valve and loosened from the bronchus. It was withdrawn in the lung slightly and fixed again at a point in the primary bronchus, so as to include in the cannulation the whole of the lower and accessory lobes. The cannula was connected to the submerged tube of the valve. With the first expiration air bubbled freely from the tube, and with inspiration water rose about $5 \mathrm{~cm}$. in the tube. The second expiration produced distinctly less bubbling, the third and fourth produced still less, and after that no more air was expelled past the valve, with the exception that one or two times when expiration was unusually forceful a bubble escaped. The inspiratory elevation of the water in the tube in- 
creased with each respiration and, after the fourth or fifth, remained at about $8 \mathrm{~cm}$. The total volume of air which had escaped at the valve was evidently no more than the amount of tidal air that the cannulated division of the lobe might be supposed to contain. The dog was sacrificed, and the position and security of the attachment of the cannula were verified.

This experiment, with the order of the maneuvers varied, was performed in many dogs, and the results were always similar to these. In one case, the air expired from a division of the lower lobe over a period of 3 hours was measured and found to amount to $4,000 \mathrm{cc}$; ; and in another case the amount was $2,700 \mathrm{cc}$. in $1 \frac{1}{2}$ hours. On the other hand, the quantity of air expired from a cannulated unit of two or three whole lobes was never greater than the volume of tidal air which it was reasonable to suppose that portion of the lungs normally possessed.

\section{Deductions}

5. After obstruction of the bronchus of a division of lobules in one pulmonary lobe in living dogs, that division may inspire and expire spontaneously and freely by another channel and maintain its inflation. The channel is probably the collateral passage, already referred to, which connects the airways of adjacent lobules. This function may be termed collateral respiration.

6. The fact that collateral respiration occurs during quiet breathing spontaneously after bronchial obstruction characterizes it as a natural function and eliminates the possibility of its occurrence as an artifact from rupture of air passages.

7. Collateral respiration does not take place between lobules which lie adjacent in different lobes $(\mathrm{dog})$.

\section{Section 4}

Object: To measure the resistance offered to collateral transfer of air between pulmonary lobules.

Experiments in vitro and in vivo, in man, dog and cat.

Protocol 8. Lungs of man. One lobe expanded in a negative pressure chamber; the bronchi of several lobules injected in turn with air; the force of inflation necessary to transfer air collaterally between lobules measured. The upper lobe was obtained from the lungs of a young. woman who had died acutely from internal abdominal injuries. The stem bronchus was found to divide into four branches outside the lobe, and the lobules which these supplied were arranged side by side as shown in the diagram, Figure 1, A, lettered $a, b, c, d$. All four were fitted with cannulas. The specimen was placed in a negative pressure chamber, with cannulas $b$ and $c$ extending to 
the outside and cannulas $a$ and $d$ closed. The lobe was then inflated to about normal expansion. When equilibrium was established (pressure of $-10 \mathrm{~cm} . \mathrm{H}_{2} \mathrm{O}$ in the chamber), cannula $b$ was connected with the necessary apparatus and injected with air, while cannula $c$ was submerged in water and watched for the escape of air, Figure 1. The pressure of injection was $3.6 \mathrm{~cm} . \mathrm{H}_{2} \mathrm{O}$ at the time that escape began, and at pressures very slightly higher than this the escape was exceedingly free. With the flow thus established the pressure of injection was lowered, and it was found that the flow continued until a pressure of $2 \mathrm{~cm} . \mathrm{H}_{2} \mathrm{O}$ was reached. The first reading was called "initiation" pressure and the second "minimum" pressure. Cannula $b$ was then closed and replaced by cannula $d$; and the latter was injected with air and cannula $c$ was observed. (Pressure of $-10 \mathrm{~cm} . \mathrm{H}_{2} \mathrm{O}$ in the chamber.) The "initiation" pressure was found to be $1.5 \mathrm{~cm} . \mathrm{H}_{2} \mathrm{O}$ and the "minimum" pressure $1.2 \mathrm{~cm} . \mathrm{H}_{2} \mathrm{O}$. Again, cannula $d$ was closed and exchanged for cannula $a$. (Pressure of $-10 \mathrm{~cm} . \mathrm{H}_{2} \mathrm{O}$ in the chamber.) Cannula $a$ was injected and $b$ observed. The "initiation" pressure was 4.0 $\mathrm{cm} . \mathrm{H}_{2} \mathrm{O}$ and the "minimum" pressure $2.8 \mathrm{~cm} . \mathrm{H}_{2} \mathrm{O}$.

This experiment was performed, also, with lung lobes from two dogs and one cat. Each lobe had two cannulas, one supplying a single lobule and the other the remaining lobules. The left lower lobe of one dog's lungs gave readings of $3.0 \mathrm{~cm} . \quad \mathrm{H}_{2} \mathrm{O}$ for "initiation" pressure and $1.4 \mathrm{~cm} . \mathrm{H}_{2} \mathrm{O}$ for "minimum" pressure. The left upper lobe of another gave $4.0 \mathrm{~cm} . \mathrm{H}_{2} \mathrm{O}$ for "initiation" pressure and $1.6 \mathrm{~cm} . \mathrm{H}_{2} \mathrm{O}$ for "minimum" pressure. The right lower lobe of the cat gave $1.6 \mathrm{~cm} . \mathrm{H}_{2} \mathrm{O}$ for "minimum" pressure.

The same experiment, without the chamber and with the lungs collapsed as from autopsy, was carried out with one specimen from man and with specimens from several dogs. The readings were consistently somewhat higher than those which have been given for expanded specimens. (Table 1.)

TABLE 1

Minimum pressure of inflation required for collateral transfer of air between pulmonary lobules in vitro

$\begin{array}{ccc}\text { Species } & \text { Lobe collapsed } & \text { Lobe expanded } \\ & \mathrm{cm} . \mathrm{H}_{2} \mathrm{O} & \mathrm{cm} . \mathrm{H}_{2} \mathrm{O} \\ \text { Man } & 7.5 & 2.0 \\ & 7.5 & 1.2 \\ \text { Dog } & 5.5 & \\ & 10.0 & 1.4 \\ \text { Cat } & 10.0 & 1.6 \\ & & 1.6\end{array}$

Protocol 9. Living dog's lungs. The bronchus of a division of lobules in one lobe obstructed with a valve, to allow only expiration for that part of the lungs: the pressures in the obstructed and free bronchi measured at inspiration; the 


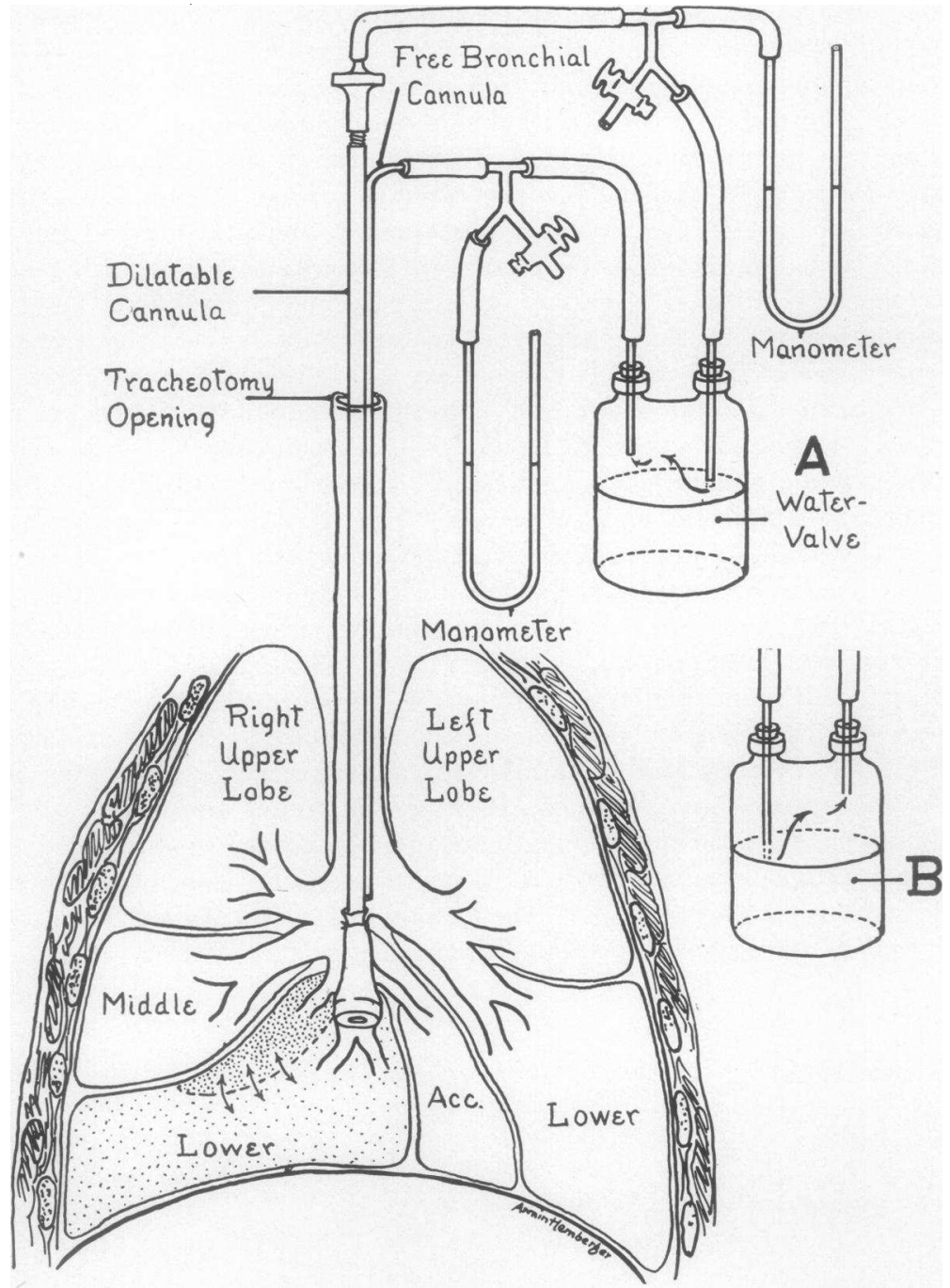

Fig. 5. Apparatus for Measuring Resistance to Collateral RESPIRATION

To the apparatus of Figure 3 have been added one free bronchial cannula with connections to the water-valve, and two manometers. 
valve reversed; the pressures in the obstructed and free bronchi measured at expiration; the forces producing collateral respiration calculated. A dog was anesthetized and tracheotomized. The dilatable cannula was introduced through the tracheotomy opening and fixed in the bronchial tree of the right lower lobe, at a point just distal to the origin of the first branch of the lobe. The cannula was connected to the submerged tube of the water-valve and to a manometer. A long slender tube was then passed beside the bronchial cannula through the trachea to the primary bronchus and its end was brought to lie freely within the lumen just proximal to the point of attachment of the cannula. This tube was connected to the elevated tube of the water-valve and to a second manometer, Figure 5, A. Now, at each expiration air bubbled past the valve (from the cannulated part of the lungs to the primary bronchus), and at each inspiration water rose a short distance in the submerged tube of the valve. This action was observed for about one-half hour and noted to be persistent. The manometers were read from time to time, and the readings were found to be stationary. Representative readings were: $1.4 \mathrm{~cm} . \mathrm{H}_{2} \mathrm{O}$ and $0.4 \mathrm{~cm} . \mathrm{H}_{2} \mathrm{O}$ at expiration, and $-1.8 \mathrm{~cm}$. $\mathrm{H}_{2} \mathrm{O}$ and $-0.6 \mathrm{~cm} . \mathrm{H}_{2} \mathrm{O}$ at inspiration. The first of each of these pairs of readings was from the manometer attached to the dilatable cannula and represented the pressure within the obstructed bronchi, and the second of each was from the manometer of the free cannula and gave the pressure within the unobstructed bronchi. The difference was taken between the two readings at inspiration, which is $1.2 \mathrm{~cm} . \mathrm{H}_{2} \mathrm{O}$, and this was interpreted to be the predominance of pressure in the unobstructed as compared to the obstructed bronchi, effecting the collateral transfer of air into the obstructed division of the lobe. Next, the water-valve was reversed, Figure 5, B. At each inspiration air bubbled past the valve (from the primary bronchus to the cannulated part of the lungs), and with expiration water rose a few centimeters into the submerged tube of the valve. This was observed for one-half hour, noted to persist, and the manometers were read from time to time. Representative readings were: $-1.2 \mathrm{~cm} . \mathrm{H}_{2} \mathrm{O}$ and -0.2 $\mathrm{cm} . \mathrm{H}_{2} \mathrm{O}$ at inspiration, and $1.0 \mathrm{~cm} . \mathrm{H}_{2} \mathrm{O}$ and $0.5 \mathrm{~cm} . \mathrm{H}_{2} \mathrm{O}$ at expiration. The order in each pair of these readings is the same as before. The difference between the two at expiration was taken, which is $0.5 \mathrm{~cm} . \mathrm{H}_{2} \mathrm{O}$, and this was interpreted to be the predominance of pressure in the obstructed as compared to the unobstructed bronchi, effecting the collateral transfer of air out of the obstructed division of the lobe. (Table 2.)

This experiment was repeated in its essential features on many other dogs and the values obtained were the same or very similar to those when the animals were breathing lightly as in this experiment. With deeper breathing the values were higher, that is, the predominances of pressure effecting collateral respiration were higher, of ten many times the values quoted. 
TABLE 2

Intrabronchial pressures produced in breathing after obstruction of a lobular bronchus in vivo

$\begin{array}{lcc}\text { Position } & \text { At inspiration } & \text { At expiration } \\ & \mathrm{cm} . \mathrm{H}_{2} \mathrm{O} & \mathrm{cm} . \mathrm{H}_{2} \mathrm{O} \\ \text { In obstructed bronchi } & -1.8 & 1.0 \\ \text { In free bronchi } & -0.6 & 0.5 \\ & \text { Difference } & \text { Difference } \\ & 1.2 & 0.5 \\ & \text { (Force producing } & \text { (Force producing } \\ & \text { collateral } & \text { collateral } \\ & \text { inspiration) } & \text { expiration) } \\ & \text { Deductions } & \end{array}$

8. The resistance offered to collateral transfer of air between pulmonary lobules in vitro is very small, particularly when the specimen is somewhat expanded during examination. It may be overcome by force as small as the weight of $1 \mathrm{cc}$. of water.

9. After obstruction of the bronchus of a pulmonary lobule in vivo, the pressures of breathing in the obstructed air passages fluctuate more widely than do the pressures in the free passages. Thus, during inspiration, the pressures in the obstructed passages fall below those in the free passages, and during expiration the former rise above the latter. These differences, or predominances, are proportional with the depth of breathing, being as low as $0.5 \mathrm{~cm} . \mathrm{H}_{2} \mathrm{O}$ pressure in very light breathing and very much greater in forceful breathing. These are evidently the forces which effect the transfer of air in collateral respiration. It may be said, therefore, that the resistance offered to collateral transfer of air between pulmonary lobules in vivo is exceedingly small, smaller than in vitro.

10. Air may be transferred collaterally between pulmonary lobules in the same lobe, even when the two concerned are separated by another lobule with obstructed bronchus. Under these circumstances, the resistance to the passages of air is somewhat increased but remains within the limits of the forces which act during quiet breathing to effect collateral respiration.

\section{Section 5}

Object: To characterize the connections by which air is transferred collaterally between pulmonary lobules.

Experiments in vitro and in vivo, in the dog. 
Protocol 10. Dog's lungs-one lobe. The bronchus of one lobule instilled with colored water (methylene blue solution); the path taken by the water observed. The right lower lobe of a dog's lungs was obtained and prepared by fixing a cannula in the stem bronchus and a second in the first bronchial branch. The cannulas were then held in the vertical position, suspending the specimen from their lower ends, and a 0.5 per cent aqueous solution of methylene blue was instilled drop by drop into the cannula of the bronchial branch, until no more would immediately enter. The lobule supplied by that branch became deeply stained. The cannula was then closed and the specimen was laid aside for one-half hour. No change was noted in the external appearance of the specimen during that time, but frothy fluid stained deep blue was found in the cannula of the stem bronchus and in the larger bronchi of the part of the lobe which it supplied. Cross-section of the lobe showed the parenchyma of the separately cannulated lobule sodden with fluid, deep blue and sharply demarkated from the parenchyma of the rest of the lobe, which was unstained.

In other experiments of this sort the stain was injected with 15 to $30 \mathrm{~cm}$. $\mathrm{H}_{2} \mathrm{O}$ pressure. In addition to immediate staining of the injected lobule, as before, these specimens showed points and areas of staining in the surfaces of the rest of the lobe within one or two minutes. Cross-section of the lobe showed solid infiltration of the injected lobule with stain and patches of staining in the parenchyma of the other parts. At the center of the patches were bronchioles filled with the stain. The line of demarcation of the injected lobule was sharp, and there was no appearance of direct extravasation from its borders. When injection was carried on a few minutes longer than this, the lobe became colored throughout with the stain.

The experiment of protocol 10 was repeated once, using a colloidal solution (5 per cent argyrol solution) for injection. Precisely the same results were obtained.

Protocol 11. Dog's lungs-one lobe. The bronchus of one lobule injected with water containing grossly discernible, solid particles (bismuth subnitrate crystals) in suspension; the path taken by the particles observed roentgenographically. The left lower lobe of a dog's lungs was obtained and prepared by tying a cannula in the first branch of the stem bronchus. An aqueous 20 per cent suspension of bismuth subnitrate (the most finely powdered commercial preparation) was injected with $10 \mathrm{~cm} . \mathrm{H}_{2} \mathrm{O}$ pressure into the cannula, and the pressure was maintained for about 15 minutes. A roentgenogram was then taken of the specimen. The pressure was raised to about $50 \mathrm{~cm}$. $\mathrm{H}_{2} \mathrm{O}$, and a second roentgenogram was made. Both negatives showed the injected lobule to be saturated and distended with the opaque mass and the rest of the lobe to be free from it.

Protocol 12. Dog's lungs-one lobe. The lobe expanded in a negative pressure chamber; the bronchus of one lobule instilled with water containing microscopic, solid particles (granules of india ink) in suspension; the path 
taken by the particles observed. The right lower lobe of a dog's lungs was obtained and prepared by fixing one cannula in the stem bronchus and another in the first bronchial branch. The specimen was then placed in the negative pressure chamber with the cannulas extending to the outside, and a moderate degree of expansion was produced by lowering the pressure in the chamber to $-10 \mathrm{~cm} . \mathrm{H}_{2} \mathrm{O}$. The chamber was then tilted onto its side, so as to bring the cannulas to a vertical position, suspending the specimen from their lower ends. Three cc. of india ink, diluted with water to 50 per cent strength, was instilled into the cannula of the bronchial branch. The pressure in the chamber was then made to fluctuate between 0 and $-10 \mathrm{~cm}$. $\mathrm{H}_{2} \mathrm{O}$ pressure four or five times, to collapse and expand the lobe alternately. Examination then showed that the cannula of the stem bronchus and the large bronchi of the part of the lobe which it supplied contained black frothy fluid.

This experiment was repeated in three other specimens, and the same results were obtained. Once, india ink was instilled into a separately cannulated lobule without the use of expansion, and the specimen was allowed to remain for 3 hours before examination. The instilled lobule was deeply and completely blackened, but no trace of ink could be found in the other parts.

Protocol 13. Living dog's lungs. The bronchus of a division of lobules in one lobe obstructed with a valve, to allow only expiration for that part of the lungs; the action of the valve observed; the pulmonary vessels to the lobe ligated; the action of the valve observed again; chloroform given for inhalation by the remainder of the lungs, and the air from the obstructed division analyzed for chloroform. A dog was anesthetized and tracheotomized. The dilatable cannula was introduced through the tracheotomy opening and fixed in the bronchial tree of the right lower lobe, at a point just distal to the origin of the first branch of the lobe. The cannula was connected to the submerged tube of the water-valve. With each expiration air passed through the valve, and with each inspiration water rose into the submerged tube. The action was observed long enough to ascertain that it was continuous. Preparation was then made to open the chest. Without disturbing the cannula, positive pressure intratracheal insufflation of air and ether-vapor was given to maintain pulmonary aeration. Incision was made through the right 5 th intercostal space, the chest was opened, and the hilus of the right lower lobe was exposed. The pulmonary artery and vein supplying that lobe were ligated securely. The chest was closed by suturing the wound, care being exercised to inflate the lungs sufficiently and thus to avoid residual pneumothorax. The positive pressure insufflation was then discontinued. It was found that the dog breathed as before and that the action of the air at the water-valve was unaltered. A series of five small bottles, each half full of water, was prepared. The rubber tube connecting the cannula to the water-valve was disconnected at the water-valve, and its end was submerged 
in the water in one of the bottles. When the air had bubbled through the water for one minute, the tube was transferred to a second bottle; and at the same time a few drops of chloroform were placed in the path of the respired air of the non-cannulated parts of the lungs. After one minute, the tube was taken to a third bottle and allowed to remain there for one minute. The two remaining bottles were used in succession in the same way. The water in the bottles was then tested for the presence of chloroform by the method of Ross (51). The first specimen was negative and the rest gave strongly positive reactions.

\section{Deductions}

11. Openings smaller in calibre than the terminal respiratory ducts and alveoli connect the arborizations of adjacent pulmonary lobules in the same lobe. This is deduced from the fact that india ink granules may pass collaterally from one lobule to another, while the larger bismuth crystals, which are readily injected through the bronchi to the alveoli, do not pass collaterally. These openings are patent only when the alveoli are expanded to some degree beyond that of the state of collapse produced by removal of the lungs from the body, since india ink easily reaches the alveoli when the lungs are collapsed but passes collaterally from lobule to lobule only with expansion of the lungs.

12. The pulmonary circulation plays no essential part in the mechanism of collateral respiration.

13. Chloroform vapor passes readily with the air during collateral respiration.

\section{Section 6}

Object: To determine the degree of interference from the presence of fluids in the bronchi with the transfer of air collaterally between pulmonary lobules.

Experiments in ritro and in vivo, in the dog.

Protocol 14. Dog's lungs-one edematous lobe. The lobe expanded in a negative pressure chamber; the bronchus of one lobule injected with air, and the force of inflation necessary to transfer air collaterally between lobules measured; w'ater injected into the same bronchus; air again injected, and the force of inflation necessary to transfer air collaterally between lobules measured. The lungs were obtained from a dog which had died from an overdose of sodium amytal. They were voluminous and heavy, and the air passages were filled with frothy fluid of pulmonary edema. The right lower lobe was detached and prepared by fixing one cannula in the stem bronchus and another in the first 
branch. The specimen was then expanded by placing it in a negative pressure chamber at $-12 \mathrm{~cm}$. $\mathrm{H}_{2} \mathrm{O}$ pressure. The cannula of the bronchial branch was connected with apparatus and injected with air, while the other was submerged in water and watched for escape of air. Escape was initiated at $2.0 \mathrm{~cm} . \mathrm{H}_{2} \mathrm{O}$ pressure. Now, $5 \mathrm{cc}$. of water was injected into the lobe through the cannula used for injection of air, and the test was repeated. The same "initiation" reading was obtained. Ten cc. of water was added; and another test gave an "initiation" value of $18 \mathrm{~cm} . \mathrm{H}_{2} \mathrm{O}$.

Protocol 15. Living dog's lungs. The bronchus of a division of lobules in one lobe obstructed with a valve, to allow only inspiration for that part of the lungs; the action of the valve observed; a colloidal solution (argyrol) injected into the cannula; the action of the valve observed again. A dog was anesthetized and tracheotomized. The dilatable cannula was introduced and fixed in the bronchial tree of the right lower lobe, at a point just distal to the origin of the first branch. The cannula was connected to the elevated tube of the water-valve, Figure 3, B. With each inspiration air bubbled past the valve (into the cannula) and with expiration water rose into the tube a few centimeters. The action was observed long enough to ascertain that it was continuous, and then $5 \mathrm{cc}$. of 10 per cent argyrol was injected into the lobe through the dilatable cannula. The action of the valve was observed again. No air passed it for the first 3 or 4 respirations, and after that the behavior was as before. Fifteen cc. of argyrol was added, and this time the valve showed no passage of air at all. The water merely rose and fell in the tube.

\section{Deductions}

14. Collateral transfer of air between pulmonary lobules is entirely prevented by the presence of large amounts of water or a colloidal fluid in the air passages. Smaller amounts interfere less and may only slightly raise the resistance; and the intrabronchial fluid in marked pulmonary edema may not cause any appreciable resistance. The smaller amounts of fluid probably become so distributed and divided among the peripheral airways by the current of air as to leave parts free for collateral transfer.

\section{DISCUSSION}

Two possible sources of error were kept particularly in mind during the performance of these experiments, namely, leakage of air at the point of attachment of the dilatable cannula to the bronchial wall, and rupture of minute airways in the lungs. These appear to have been eliminated satisfactorily. First, leakage seemed highly improbable to all observers of the action of the dilatable cannula and of the 
rigorous tests to which its attachment was submitted; but still more convincing are the facts that the appearances of collateral transfer always failed to occur with the cannula fixed in the primary bronchi, and that in experiments in vitro, with the cannulas tied in the bronchi, collateral transfer was obtained as well. Second, the extremely low pressures of injection used in producing the transfer artificially and the fact that the transfer is initiated and maintained spontaneously in vivo during quiet breathing appear to remove the possibility of alveolar rupture as an essential part of the process.

Collateral transfer of air in the lungs requires intimate fusion of the lobules, and it is prevented by the presence of complete septa between the lobules. These circumstances have been found to vary considerably with different species and with different regions of the lungs. In man, as, also, in the dog and cat, the interlobular septa are thin and incomplete, while the interlobar septa are thick and complete. In these species air was found to pass freely from lobule to lobule only when they lay within the same lobe. Tests were applied to the major lobules alone, but it is likely that collateral transfer is possible also between minor lobules, considering the similarity of the two as to degree of fusion. Technical limitations prevented as extensive examination for collateral respiration in living animals as was applied for the transfer of air in specimens removed from the body, but it appears very likely that collateral respiration occurs in all parts of the lungs where collateral transfer of air was found possible.

The means by which air passes collaterally from lobule to lobule remains in doubt. Some light is thrown upon this by the behavior of gases, liquids, and particulate matter injected into the air passages of a lobule. We conclude that anatomical openings must exist between the lobules at their planes of fusion, that the openings lie between the terminal airways, perhaps between the alveoli, that they are extremely minute in caliber, smaller than the alveoli, and that they are patent only when the parenchyma is expanded. Of the structures of the lung with which we are acquainted, the pores of Kohn meet these criteria best. If these pores are normal structures, they may well account for all of the appearances of collateral transfer. We are inclined to believe that collateral transfer of air depends also to some extent upon diffusion. 
From the evidence at hand as to the minuteness of the openings between the lobules, one readily understands the reason for the negative results of the anatomical investigations of many workers as to the question of interlobular anastomosis. Whatever the openings may be, it is clear that they permit only very limpid fluids to pass. The masses which investigators have injected in making corrosion preparations have been far too viscid. Penetration of the injected material to the alveoli cannot be taken as adequate for the demonstration of interlobular connections-witness our negative results with bismuth. Moreover, the practice has been to inject the whole specimen at once from the stem bronchus, rather than to inject the bronchus of one lobule at a time; and this afforded poor opportunity for the masses to flow from lobule to lobule. Also, wax models of the lungs cannot be expected to demonstrate the presence of such minute structures as are the openings in question, for microsections cannot be cut thin enough.

The term, collateral respiration, is applied to the function described in this paper, because of the analogy which it bears to collateral circulation by capillary anastomosis in the blood-vascular system. The two functions are fairly similar in mechanism of operation and in physiological purpose.

Collateral respiration, like collateral circulation, depends for its occurrence upon the development of differences of pressure in two adjacent units of passages, and this takes place only when the main channel of supply for one unit is obstructed. The differences of pressure in the bronchial tree, between obstructed and free parts, have been measured directly in these experiments and were found to vary in magnitude with the depth of breathing. Only very slight differences, such as occur in light breathing, are required to carry on collateral respiration.

The physiological purpose of collateral respiration becomes apparent in reviewing the work in bronchial obstruction which has already been reported by members of our group. Collateral respiration has much the same economic rôle in the operation of the bronchial tree as collateral circulation has in that of the vascular system, for it acts to conserve the function of parts which have become obstructed. The small bronchial branches are obstructed much more frequently than are the blood vessels, in all probability, since occluding substances, especially products of inflammation, find very ready access to the air 
passages. Furthermore, the ebb-and-flow principle of aeration of the lungs is at a greater mechanical disadvantage in the presence of obstruction by fluids than is circulation. Thus, it is a matter of common observation in clinical fluoroscopy of the lungs, with lipiodol in the bronchi for diagnostic purposes, that a column of lipiodol in a small bronchus remains there during respiration, rides to and fro, and permits no air to pass. Its expulsion occurs only with forceful expiratory effort (cough). No more than a droplet of fluid is required to obstruct a capillary bronchiole in this way, and, if it occurs from bronchial secretion at night or during any period when cough is absent for some time, the obstruction must remain and the imprisoned air undergo absorption. It is here that collateral respiration probably functions to maintain the air supply, until cough or another eliminative force may act. Experiments with dogs by Lindskog and Van Allen (52) have shown that air may be absorbed from an obstructed lung lobe (incapable of collateral respiration) in sufficient quantity within 30 minutes to render cough entirely ineffectual for elimination. Under these circumstances, too, the lobe becomes atelectatic as a rule within 24 hours. Van Allen and Adams (53) and Van Allen and Lindskog $(49,50)$ have found that the bronchus of one lobular division in a dog's lungs may be obstructed and remain fully air-containing for indefinitely long periods of time. Adams (54) has repeated and subsequently confirmed this finding. The lungs of man with chronic destructive diseases are being searched at autopsy for the presence of bronchiolar stenosis by Van Allen and Ch'in. In the first specimen examined there was found occlusion of a tertiary bronchus at one point by a tuberculous lesion, with the lobule, which was supplied by the bronchus, air-containing. Our experience both in man and in laboratory animals has been that lobular atelectasis develops after obstruction when, in addition to occlusion of the bronchus of the lobule, there is occlusion of the airways at the periphery of the lobule upon which collateral respiration depends.

\section{SUMMARY}

The air-tightness of the pulmonary lobule has been investigated in several species of animal, including man, in order to determine whether air may pass collaterally from lobule to lobule. This is found to be the 
case in those species and in those parts of the lungs where the lobules are intimately fused and lack complete interlobular septa. In man, collateral transfer of air between lobules is possible where the lobules belong to the same lobe, and not where they belong to different lobes. The transfer is very free. Thin liquids and finely particulate matter are also able to pass. The mode of transfer of air has not been surely determined; but it is supposed to depend both upon diffusion through the alveolar walls separating the lobules and upon flow through minute openings in these membranes.

After obstruction of the bronchus of one lobular division of a lobe in the lungs of living dogs, air is found to enter and to leave that part during the breathing by means of collateral connections with adjacent unobstructed lobules. To this function of the obstructed pulmonary lobule the term collateral respiration is given. It fails to occur between lobules situated in different lobes.

The physiological significance of collateral respiration is discussed in its relation to bronchial obstruction and pulmonary atelectasis.

\section{BIBLIOGRAPHY}

1. Van Allen, C. M., Lindskog, G. E., and Richter, H. G., Yale J. Biol. and Med., 1930, ii, 297. Gaseous Interchange Between Adjacent Lung Lobules.

2. Zimmermann, quoted from Flint (6).

3. Hansemann, quoted from Flint (6).

4. Merkel, quoted from Flint (6).

5. Schulze, quoted from Flint (6).

6. Flint, J., Am. J. Anat., 1906, vi, 1. The Development of the Lungs.

7. Miller, quoted from Flint (6).

8. Laguesse and d'Hardiviller, quoted from Flint (6).

9. Oppel, quoted from Flint (6).

10. Miller, W. S., J. Exp. Med., 1925, xlii, 779. The Alveolar Pores of Pneumonia.

11. Macklin, C. C., Physiol. Rev., 1929, ix, 1. Musculature of the Bronchi and Lungs.

12. Hales, S., Statistical Essays Containing Vegetable Staticks or an Account of Some Statical Experiments on the Sap in Vegetables and also a Specimen of an Attempt to Analyse the Air: London, W. Innys and R. Manby, 1733, ii, 2nd Ed., 160.

13. Ewald, J. R. and Kobert, R., Arch. f. d. ges. Physiol., 1883, xxxi, 160. Ist die Lunge luftdicht? 
14. Van Allen, C. and Johnson, C. Unpublished work.

15. Joannides, M. and Tsoulos, G. D., Arch. Surg., 1930, xxi, 333. The Etiology of Interstitial and Mediastinal Emphysema.

16. Bichat, quoted from Beneke, R., Verhandl. d. dtsch. path. Gesellsch., 1913, xvi, 263. Über Luftembolie im grossen Kreislauf.

17. Iversen, A., quoted from Walcher, K., Mitt. a. d. Grenzgeb. d. Med. u. Chir., 1926, xxxix, 314. Ueber die Luftembolie.

18. Peltauf, quoted from Walcher, K., Mitt. a. d. Grenzgeb. d. Med. u. Chir., 1926, xxxix, 314. Ueber die Luftembolie.

19. Lindblom, A., Virchow's Arch., 1924, cclii, 197. Über Luftembolie bei Neugeborenen und Saüglingen und die gerichtlich medinizinsche Bedeutung derselben.

20. Neubürger, K., Klin. Wchnschr., 1925, iv, 113. Über die Pathogenese der Keuchhusten-eklampsie.

21. Husler, J. and Spatz, H., Ztschr. f. Kinderh., 1924, xxxviii, 428. Die "Keuchhusten-Eklampsie."

22. Sauerbruch, F., Chirurgie der Brustorgane. Berlin, Julius Springer, 1925, ii, 2nd Ed., 160.

23. Kohn, H., München. med. Wchnschr., 1893, xl, 42. Zur Histologie der indurirenden fibrinösen Pneumonie.

24. Schultz, quoted from Müller (43).

25. Kölloker, quoted from Müller (43).

26. Water, quoted from Müller (43).

27. Ribbert, quoted from Müller (43).

28. v. Ebner, quoted from Müller (43).

29. Laguesse and d'Hardiviller, quoted from Müller (43).

30. Miller, quoted from Müller (43).

31. Malpighi, quoted from Müller (43).

32. Henle, quoted from Müller (43).

33. Adrian, quoted from Müller (43).

34. Hauser, quoted from Müller (43).

35. Zimmermann, quoted from Müller (43).

36. Hansemann, quoted from Müller (43).

37. Sudruski, quoted from Müller (43).

38. Linser, quoted from Müller (43).

39. Sobotta, quoted from Müller (43).

40. Nicolas, quoted from Müller (43).

41. Merkel, quoted from Müller (43).

42. Stöhr, quoted from Müller (43).

43. Müller, J., Arch. f. mikr. Anat., 1907, Ixix, 1. Zur vergleichenden Histologie der Lungen unserer Haussäugetiere.

44. Ogawa, C., Am. J. Anat., 1920, xxvii, 333. Contributions to the Histology of the Respiratory Spaces of Vertebrate Lungs.

45. Van Allen, C. M., Yale J. Biol. and Med., 1930, ii, 295. A Dilatable Bronchial Cannula. 
46. Guedel, A. E. and Waters, R. M., Anesth. and Analg., 1928, vii, 238. A New Intratracheal Catheter.

47. Coryllos, P. and Birnbaum, G. L. (Trans. Am. Assoc. Thorac. Surg.), Arch. Surg., 1930, xxi, 1214. Alveolar Gas Exchanges and Atelectasis. The Mechanism of Gas Absorption in Bronchial Obstruction.

48. Feiermann, I. M., Arch. f. klin. Chir., 1930, clix, 236. Einfluss der Thorakoplastik auf die Atmung.

49. Van Allen, C. M. and Lindskog, G. E. (Trans. Am. Assoc. Thorac. Surg., May 15, 1930), Arch. Surg., 1930, xxi, 1195. Obstructive Pulmonary Atelectasis, Problems of Pathogenesis and Clinical Management.

50. Van Allen, C. M. and Lindskog, G. E., Surg., Gynec. and Obst., 1931, liii, 16. Collateral Respiration.

51. Ross, J. H., J. Biol. Chem., 1923, 1viii, 641. A Color Test for Chloroform and Chloral Hydrate.

52. Lindskog, G. E. and Van Allen, C. M. The Aerodynamics of Bronchial Obstruction. Arch. Surg. (In press.)

53. Van Allen, C. M. and Adams, W. E., Surg., Gynec. and Obst., 1930, i, 385. The Mechanism of Obstructive Pulmonary Atelectasis.

54. Adams, W. E., Proc. Soc. Exp. Biol. and Med., June 1930, xxrii, 982. Further Studies in Obstructive Pulmonary Atelectasis. 